\title{
PERSPECTIVE
}

\section{Multi-stability and the origin of microbial community types}

\author{
Didier Gonze ${ }^{1}$, Leo Lahti ${ }^{2,3,4}$, Jeroen Raes $^{2,3}$ and Karoline Faust ${ }^{2}$ \\ ${ }^{1}$ Department of Chemistry, Unité de Chronobiologie Théorique, Faculté des Sciences CP 231, Université Libre \\ de Bruxelles, Bvd du Triomphe, 1050 Bruxelles, Belgium; ${ }^{2}$ Department of Microbiology and Immunology, \\ Rega Institute, KU Leuven, Campus Gasthuisberg, Leuven, Belgium; ${ }^{3}$ VIB Center for Microbiology, Campus \\ Gasthuisberg, Leuven, Belgium and ${ }^{4}$ Department of Mathematics and Statistics, 20014 University of Turku, \\ Finland
}

\begin{abstract}
The study of host-associated microbial community composition has suggested the presence of alternative community types. We discuss three mechanisms that could explain these observations. The most commonly invoked mechanism links community types to a response to environmental change; alternatively, community types were shown to emerge from interactions between members of local communities sampled from a metacommunity. Here, we emphasize multi-stability as a third mechanism, giving rise to different community types in the same environmental conditions. We illustrate with a toy model how multi-stability can generate community types and discuss the consequences of multi-stability for data interpretation.
\end{abstract}

The ISME Journal (2017) 11, 2159-2166; doi:10.1038/ismej.2017.60; published online 5 May 2017

\section{Introduction}

In the past decade, the microbial composition of a large number of samples from different environments was determined. For host-associated microbiota in particular, samples can be grouped into clusters based on their microbial composition. For instance, samples of the vaginal microbiome were found to form five distinct clusters, four of them dominated by different Lactobacillus species and the fifth of mixed character (Ravel et al., 2011). Clusters were also reported for the gut microbiome, where they are known as enterotypes (Arumugam et al., 2011). A recent clustering analysis suggests that oral microbiota can be likewise divided into clusters (Ding and Schloss, 2014).

Figure 1a illustrates clusters present in the Flemish Gut Flora Project data (Falony et al., 2016), one of the biggest gut data sets available to date (1106 samples). The clusters are visualized as mountains in a landscape plot, where peaks are the higher the more samples share the same community composition. We refer to both distinct and overlapping clusters as community types. In the following, we

Correspondence: K Faust, Department of Microbiology and Immunology, Rega institute, Bus 1028, Campus Gasthuisberg, Herestraat 49, Leuven 3000, Belgium.

E-mail: karoline.faust@kuleuven.be

Received 6 December 2016; revised 28 February 2017; accepted 10 March 2017; published online 5 May 2017 will discuss different mechanisms that could explain alternative community types.

\section{Mechanisms behind alternative community types}

The most frequently mentioned mechanism is a continuous response of the community to a changing environmental parameter (Figure 1b). If the response is gradual, many transitional community configurations can be observed, resulting in a gradient. In contrast, if the response is abrupt, the probability of detecting transitional community configurations decreases, such that two distinct community types emerge: one before and the other after the environmental change. This mechanism can be implemented with the generalized Lotka-Volterra model. In its standard form, the generalized Lotka-Volterr model describes community dynamics as a function of growth rates and interaction strengths between community members. The generalized LotkaVolterr model has been extended with a susceptibility term, which models how each species responds to a perturbation (Stein et al., 2013). This susceptibility term can also capture the effect of environmental change on the community.

Recently, a novel community-type generating mechanism was proposed (Gibson et al., 2016) (Figure 1c). Gibson and colleagues link the emergence of alternative community types to a strong heterogeneity of interaction strengths or alternatively to the 

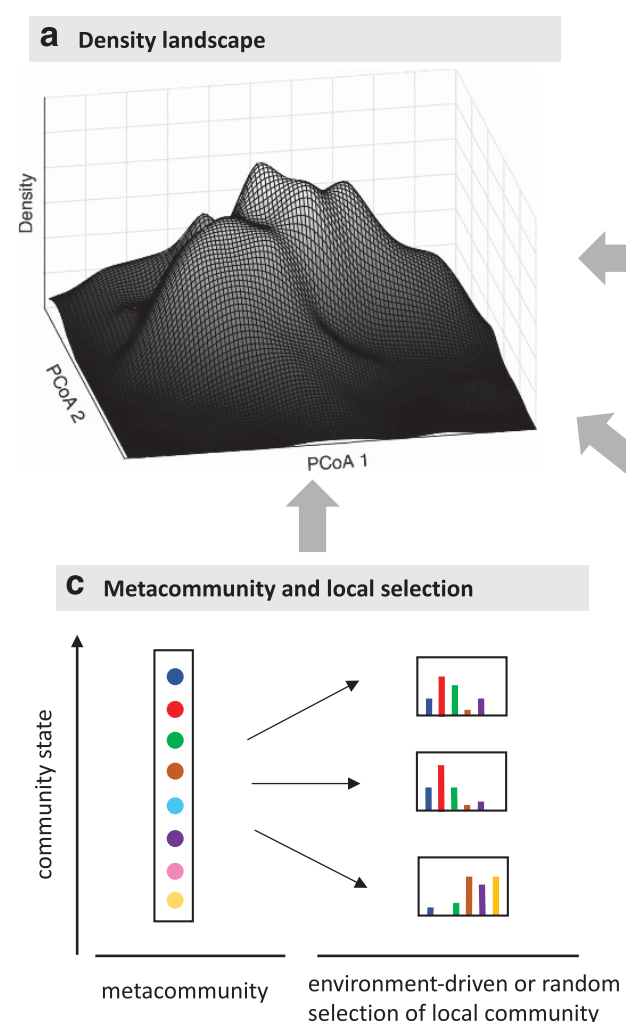

b Continuous response to environment

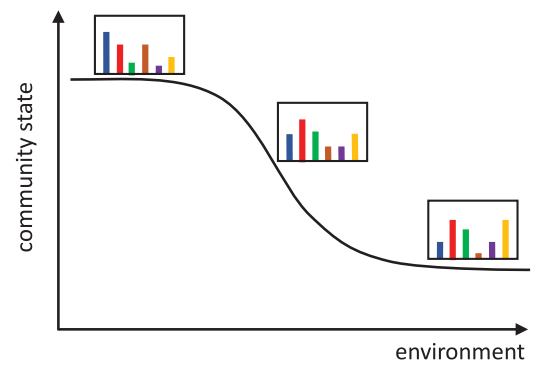

d Multi-stability and hysteresis

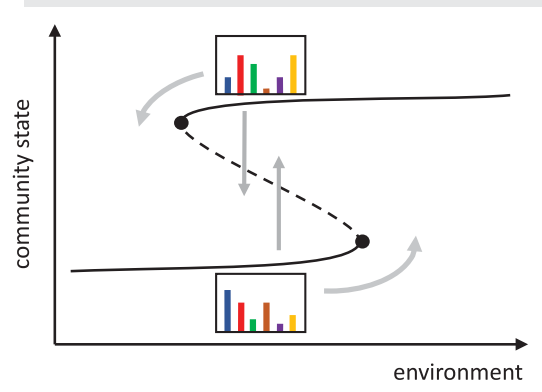

Figure 1 Overview of different mechanisms generating community types. (a) In general, peaks in the landscape of possible community configurations are interpreted as community types. The landscape plot shown here was generated with data from the Flemish Gut Flora Project. It combines a principal coordinates analysis plot, which summarizes community composition in two dimensions, with a density landscape. The latter encodes the frequency of observed community configurations as height. The mountain peaks thus represent alternative community types. The exact mechanism behind these peaks is unknown. (b) The most frequently evoked mechanism for different community types is a continuous response to environmental change. The community type is here represented by a bar plot that depicts the abundance of different species (indicated with colored bars). The probability of detecting different community types depends on the slope of the community response curve. If the slope is steep, transitional community configurations will be harder to find, resulting in separate clusters. (c) Gibson and colleagues recently proposed a model that generates different local communities from a metacommunity through random selection. The local communities differ when interaction strengths between community members are strongly heterogeneous. The model can be adapted to describe the impact of the environment by replacing random selection with habitat filtering. (d) Finally, a multi-stable community can adopt different stable states in the same environmental conditions. A community type switch can be triggered either by adding or removing individuals belonging to species present in the community (vertical arrows) or by changing the environment (bent arrows). However, once the system is pushed across a tipping point (black point), it does not return when original conditions are restored. Instead, it has to be altered beyond another tipping point to go back to its original state. This effect is known as hysteresis.

presence of strongly interacting species (SIS). The members of local communities are sampled randomly from the metacommunity. The local community type is then defined by the particular combination of SIS present. In the absence of SIS, the dynamics between local communities is very similar; community types vanish. Two aspects of this community model are especially noteworthy: first, the particular SIS combination assigned to each local community is random. Thus, environmental differences between local communities do not explain differences in community composition, although one could introduce them easily through non-random selection of species from the metacommunity. Second, the authors found that the interaction strength heterogeneity determines the distinctness of community types. Thus, depending on parameter settings, the same mechanism generates gradients or clusters.
In the model by Gibson and colleagues, alternative communities are composed of different species subsets. However, it is possible to generate alternative community types with the same set of species. Multi-stable ecosystems have more than one stable state in the same conditions. The association between precipitation levels and tree cover is a well-established example from macroecology, where two alternative states of tree cover, corresponding to rainforest and savanna, can exist at similar precipitation levels (Hirota et al., 2011). When an environmental parameter is changed beyond the so-called tipping point, such systems respond with an abrupt switch from one stable state to another. However, since more than one stable state is present, the former community state cannot be regained by simply reversing the environmental parameter. Instead, the environmental parameter has to be 
changed beyond another tipping point (Figure 1d). This effect, known as hysteresis, introduces a path dependency: previous parameter values and community states matter. Depending on its history, the community may adopt one or another distinct state in the same environmental conditions. The importance of multi-stability was recognized early on in ecology (for example, (Lewontin, 1969; May, 1977)) and experimental evidence for it has since been found in natural systems, mesocosms and in vitro (reviewed in (Schröder et al., 2005)). Bucci and colleagues also proposed a model describing a bi-stable system of antibiotic-tolerant and sensitive gut bacteria (Bucci et al., 2012). Despite its recognized importance in other fields, the role of multistability is rarely considered in the current debate on alternative microbial community types detected in recent sequencing data sets (a notable exception is (Shetty et al., 2017)).

The mechanisms discussed above should not be confounded with ways to manipulate microbial communities. Costello et al. (2012) previously pointed out two such ways, namely (i) directly via the addition or removal of individual community members or (ii) indirectly by changing environmental parameters, which then favor different community members. Both factors may simultaneously contribute to each of the three mechanisms, but their consequences depend on the mechanism involved. Thus, the knowledge of the mechanism underlying community types will also ease their manipulation.

\section{Multi-stability: a proof-of-concept}

In the following, we demonstrate with a threespecies toy model how multi-stability generates alternative community types. Multi-stability requires interactions between species, more specifically the presence of a positive circuit (Thomas's conjecture, (Thomas and D'Ari, 1990)). Such circuits can be achieved for example through mutual inhibition between two species, as demonstrated by the genetic toggle switch (Gardner et al., 2000). Following this principle, we built a model in which three species inhibit each other, such that one species dominates while the other two are maintained at low abundance due to the repression by the dominant species (Figure 2a). The inhibition, mediated for example through exchange of (toxic) compounds or competition, is described by decreasing sigmoidal Hill functions (Figure $2 \mathrm{~b}$ and Supplementary Material). The inhibition coefficients $K_{i j}$ encode the strength of the inhibition of species $i$ by species $j$. All inhibition coefficients together form the inhibition matrix $\boldsymbol{K}$. Of note, the inhibition is the stronger the smaller the inhibition coefficient.

Depending on the initial abundances, either one of the three species dominates the community
(Figure 2c). Importantly, in the simulations, the model parameters are not altered; a change in initial abundances is sufficient to reach another community state, demonstrating the presence of three alternative stable states in the system. This multi-stability is also clearly seen in the bifurcation diagrams shown in Figure 2d. A bifurcation diagram displays, as function of a control parameter, all possible states of a dynamical system as well as their stability. In our example, the control parameter is the growth rate of species 1 (blue), which may change in response to environmental conditions such as nutrient concentration or $\mathrm{pH}$. The state of the dynamical system is defined by the abundances of the three species. The thick color curves in Figure 2d denote stable states, whereas dashed curves represent unstable states. Two low-abundance stable states and one highabundance stable state are visible, which coexist in the region highlighted by the orange background (tri-stability).

Switches from one community to another can be induced by changing the control parameter beyond the boundary of the tri-stability region. This is demonstrated in Figure 2e, where a pulse perturbation temporarily lowers the growth rate of the blue species below the boundary of the tri-stability region. In consequence, a switch from blue to green species dominance occurs. Crucially, the green species continues to dominate the community even after the end of the perturbation. A second perturbation, which increases the growth rate of the blue species above its original value (before the first perturbation), is needed to return to blue dominance, highlighting hysteresis. In real microbial communities, a variety of environmental factors could act as pulse perturbations, for example rain in soil communities, particular food components, such as fibers in gut communities and menstruation in vaginal communities.

Our model can be generalized to larger communities. As a proof-of-concept, we model a community of 15 species, which form three groups with strong inter-group inhibition and weak intra-group inhibition (Figure 3a). The tri-stable community is dominated by either one of its three groups depending on initial species abundances (Figure 3b). Interestingly, like the model of Gibson and colleagues, our proofof-concept model generates distinct clusters or gradients, depending on the inter-group interaction strengths (Figure 3c). Next, we generated a random inhibition matrix with a strongly heterogeneous distribution of interaction strengths (Figure 3d, inset). When carrying out simulations with this inhibition matrix and varying initial abundances, we detect distinct sample groups representing alternative stable states (Figure 3d). Temporarily increasing the growth rate of a SIS induces a community switch, which can only be reversed when this growth rate is lowered below its initial value (Figure 3e). 


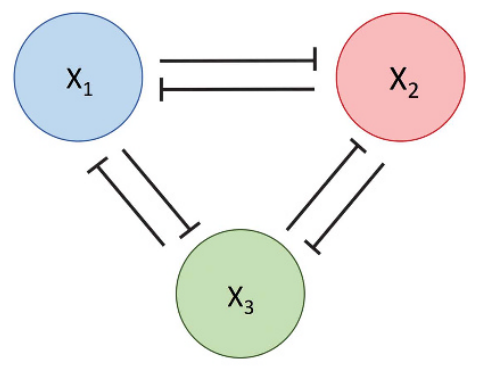

C
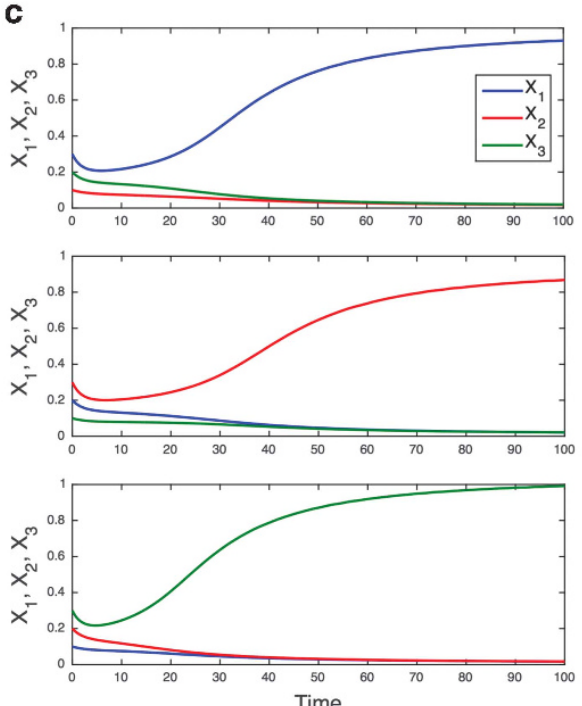

b

$$
\begin{aligned}
& \frac{d X_{i}}{d t}=X_{i}\left(b_{i} f_{i}\left(\left\{X_{k}\right\}\right)-k_{i} X_{i}\right) \\
& f_{i}\left(\left\{X_{k}\right\}\right)=\prod_{\substack{k=1 \\
k \neq i}}^{N} \frac{K_{i k}^{n}}{K_{i k}^{n}+X_{k}^{n}}
\end{aligned}
$$

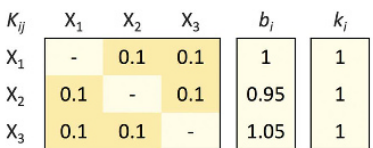
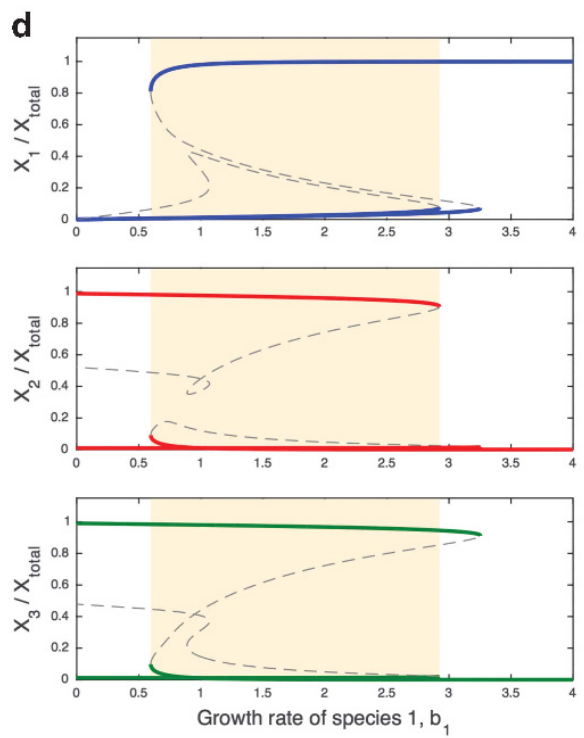

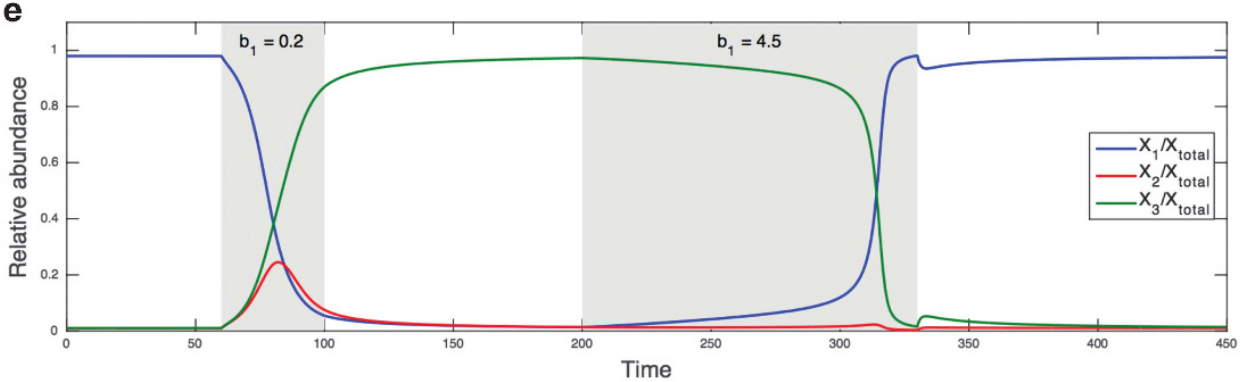

Figure 2 Toy model for multi-stable communities. (a) The toy model community consists of three species, each of which competes with the other two. (b) The toy model describes the dynamics of each species as a function of its growth rate $b_{i}$, its death rate $k_{i}$ and an inhibition term $f_{i}$. The inhibition term models how all species lower the species' growth rate using a Hill function, which takes inhibition coefficients $K_{i j}$ and the Hill coefficient $n$ (here $n=2$ ) as parameters. The interaction coefficients form a matrix, the values of which are shown. The smaller the inhibition coefficients, the stronger the inhibition. (c) Numeric simulation of the model demonstrates that three stable states exist, which depend on the initial abundances of the species. (d) The three bifurcation diagrams show for each species the range of the three stable states. (e) When perturbing the system by temporarily decreasing the growth rate of $b_{1}$ below the first tipping point, the green species replaces the blue one as the dominant species in the system. A second perturbation, increasing the growth rate of the blue species beyond the second tipping point, is needed to return to the original state. More details are provided in the Supplementary Material.

\section{Implications of multi-stability}

Knowing whether a community under study is multistable or not has important implications for the interpretation of microbial sequencing data. If a host-associated microbial community is multi-stable, it is not necessarily meaningful to explain differences in microbial composition across hosts with differences in host physiology, life style, genetics or other properties, since alternative stable states may be the result of a past perturbation rather than a current difference in host properties. Thus, the generative mechanisms underlying community types are of great relevance for understanding community variation.

Multi-stability has also implications when determining whether community dynamics is universal. The question about the universality of community dynamics was recently raised by Bashan and colleagues, who also presented a method to address 
a

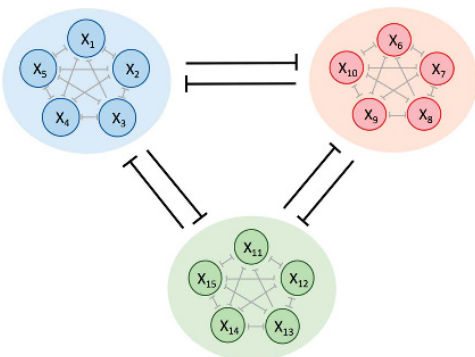

b
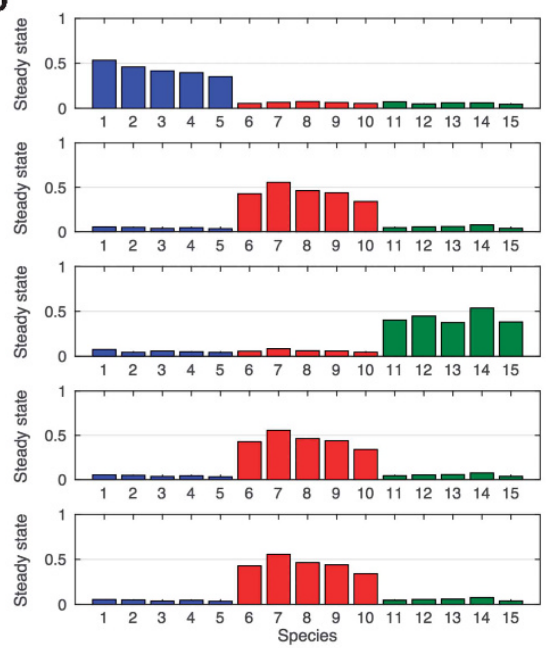

c
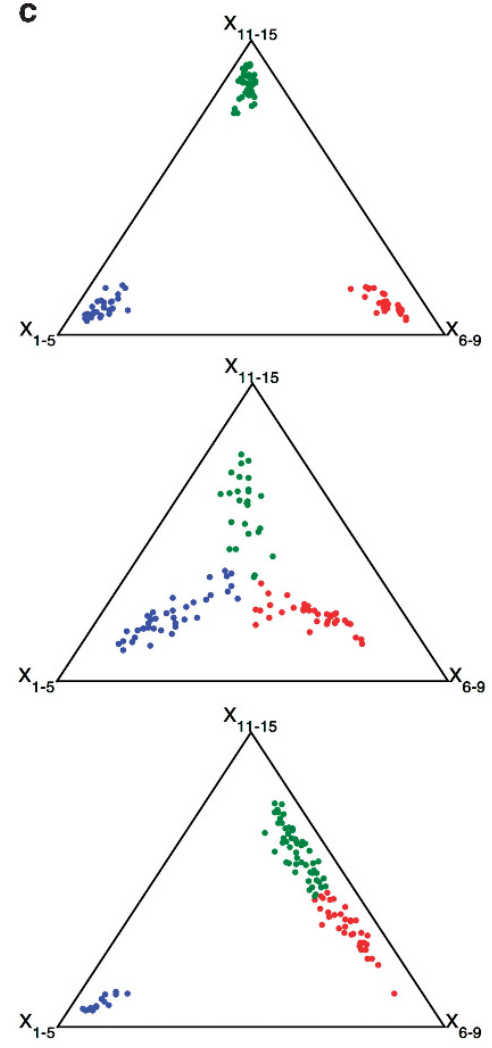
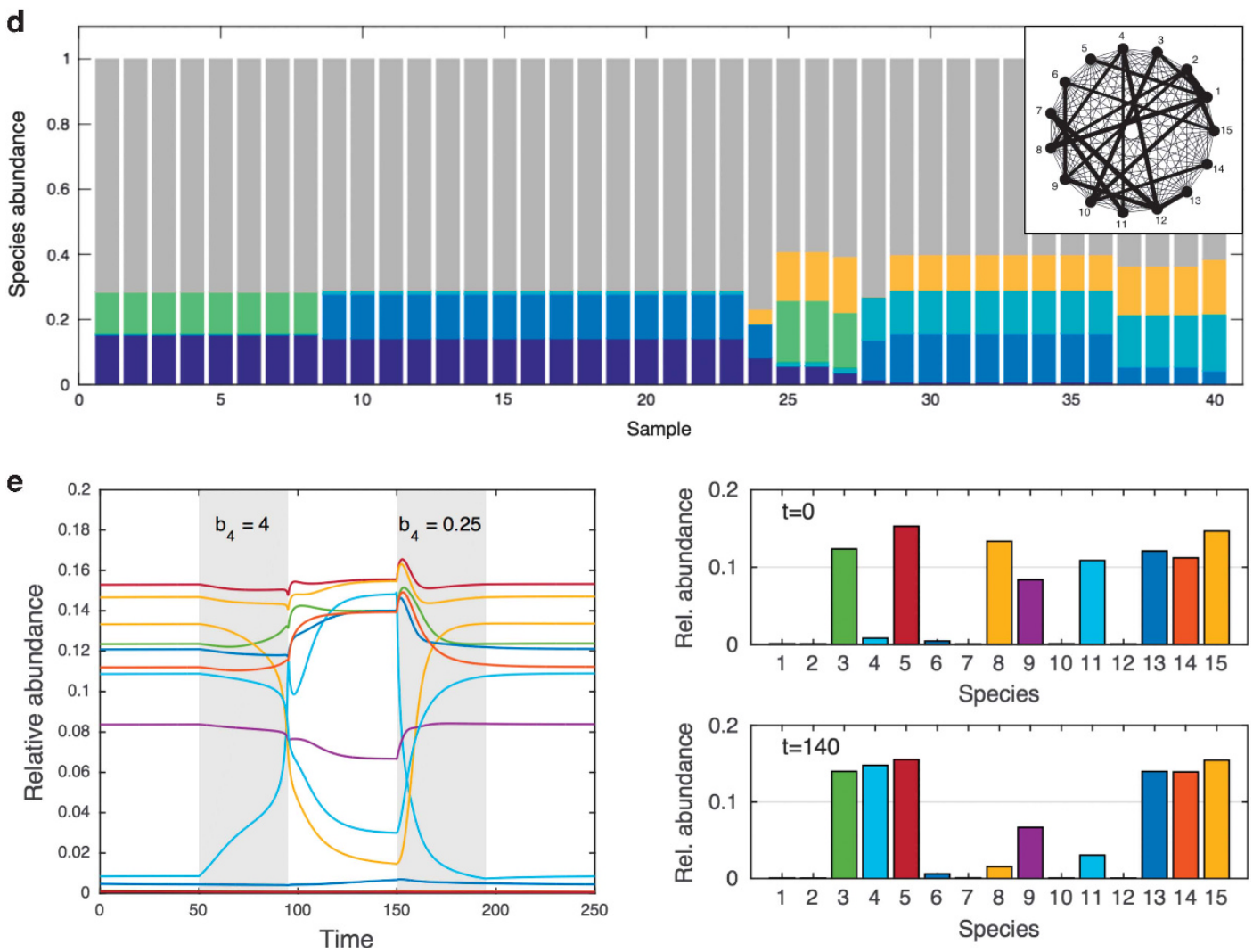

Figure 3 For caption see next page. 
it with microbial abundance data (Bashan et al., 2016). Their dissimilarity-overlap-curve method assumes that species following the same dynamics end up with the same abundance profiles, an assumption that is violated by multi-stability. Thus, multi-stable communities may well follow a universal dynamics despite being classified as nonuniversal by the dissimilarity-overlap-curve method.

Finally, the presence of multi-stability strongly questions community neutrality. According to the neutral model (Rosindell et al., 2011), the community dynamics is entirely stochastic and species interactions do not matter. Since according to Thomas's conjecture (Thomas and D'Ari, 1990), the presence of a positive circuit is a necessary condition for multi-stability, multi-stability implies the presence of species interactions and thus non-neutral community dynamics.

\section{Evidence for multi-stability}

Microbial abundance data alone are not sufficient to differentiate between possible mechanisms. If environmental data are available, then a strong association to an environmental parameter may point to the first mechanism (that is, continuous response to environmental change). For instance, the enterotypes have previously been linked to diet (Wu et al., 2011), inflammation (Chatelier et al., 2013) and stool consistency/transit time (Falony et al., 2016). Yet, in another study, high-or-low (bimodal) abundance patterns of selected gut species could not be explained by differences in diet or other metadata and, supported by additional longitudinal analysis, were interpreted as indicative of bistability (Lahti et al., 2014). Bimodal distributions were also observed in vaginal microbiota (Faust et al., 2015). However, bimodality alone is not sufficient to prove multistability. As mentioned by Scheffer and colleagues (Scheffer et al., 2009), a non-multi-stable system may respond to environmental change in a strongly nonlinear manner, leading to abrupt community change and thus bimodality. The difficulty of distinguishing true multi-stability from a response to potentially subtle environmental change has been discussed in depth before (Connell and Sousa, 1983). To identify multi-stability, Scheffer and colleagues suggest to look for early warning signs, which increase near the tipping point (Scheffer et al., 2009). The recent analysis of an artificial oral microbiome subjected to repeated sucrose pulses revealed a characteristic early warning sign, namely the critical slowing down of $\mathrm{pH}$ recovery, which ultimately led to an abrupt change of the ecosystem (Koopman et al., 2015). However, early warning signs are only applicable to systems exposed to a changing environmental parameter or system variable (for example, $\mathrm{pH}$ in (Koopman et al., 2015)) and require long time series. To overcome this limitation, Lahti and colleagues recently proposed to aggregate multiple short time series across many individuals to quantify resilience in bacterial abundance profiles and thus obtain an indicator of bistability (Lahti et al., 2014). With this indicator, the Prevotella genus was identified to exhibit alternative states of low and high abundance in the human gut.

A telltale sign of multi-stability is hysteresis, that is, the failure to return to the original community state once the original conditions are restored after a perturbation. Hints of hysteresis are seen in otherwise stable vaginal microbiota that switch from one dominant Lactobacillus species to another upon perturbation in form of menstruation or sexual intercourse in a few women (Gajer et al., 2012). Incomplete recovery, for example of gut communities exposed to antibiotic treatment (Dethlefsen and Relman, 2011), likewise may point to multi-stability. These observations are however insufficient to conclude that these microbiota are multi-stable.

Although there is to our knowledge no conclusive experimental evidence yet for multi-stability as a mechanism behind alternative microbial communities, the indications justify a deeper investigation.

\section{Discussion}

In recent years, the interpretation of alternative community types in the gut (enterotypes) has been hotly debated. While some authors interpret them as gradients or as artifacts resulting from gradients of dominant gut organisms (Knights et al., 2014;

\footnotetext{
Figure 3 Proof-of-concept model for multi-stable communities. (a) The first version of the proof-of-concept model consists of three groups of five weakly interacting species $\left(n=2, K_{i j} \approx 1\right)$. Each group strongly inhibits the other two groups $\left(n=2, K_{i j} \approx 0.5\right)$. (b) There are three stable states, each dominated by either the blue, red or green group. Each panel shows the stable distribution of the 15 species for randomly sampled initial abundances. (c) When carrying out simulations with slightly varying growth rates and inhibition coefficients and plotting resulting total group abundances together in a ternary plot, the group structure is clearly visible (upper triangle plot). However, when the inter-group inhibitions are lowered, the distinction between groups lessens (middle triangle). A gradient between two groups can also emerge if their mutual inhibition is lower than their inhibition with the third group (bottom triangle). (d) We also explored a version of the proof-of-concept model without pre-defined group structure, where inhibition coefficients were sampled from an exponential distribution and the Hill coefficient $n$ was set to 4 (inset). When repeating simulations with varying initial abundances and visualizing stable state abundances in a bar plot, distinct groups emerge. The bar plot only colors abundances of the five species that vary the most strongly across groups, whereas more homogeneous species are uniformly colored in gray. (e) As in the toy model, a community type switch can be induced by temporarily increasing the growth rate of a species (species 4 here, cyan curve). The two bar plots depict the abundance distribution before and after community type switching. Since species 4 and 8 inhibit each other, the abundance of species 8 is low in the new stable state (lower bar plot) as compared to the first stable state (upper bar plot). Hysteresis is demonstrated by the fact that the growth rate of species 4 has to be reduced below its original value to return to the original state. More details are provided in the Supplementary Material.
} 
Gorvitovskaia et al., 2016), others describe them as peaks in the landscape of all possible community configurations (Falony et al., 2016; Figure 1a). In the context of this debate, it is worthwhile to point out that each of the three mechanisms can generate gradients or clusters, depending on model parameters. Although it is important to discuss how to interpret community types, we think that the current gradient-versus-cluster debate obscures the more interesting question about which mechanism explains these patterns.

Here, we treated each mechanism separately, but it is likely that a combination of them determines community dynamics in microbial ecosystems. For instance, enterotypes may represent alternative stable states of a multi-stable system in healthy persons, but dysbiotic communities in Crohn's disease or ulcerative colitis may be driven by a strong change in environmental conditions (for example, inflammation). In addition, enterotypes may differ in their resistance to environmentally induced community shifts (Vieira-Silva et al., 2016).

In the metacommunity model as well as in our proof-of-concept model, states emerge through a few strong interactions. Thus, according to both models, the SIS, but not necessarily the most abundant species, determine the community type. Gibson and colleagues point out the special interest of SIS as targets of community engineering efforts. These species can be considered as levers with which to switch the community from one type to another. In simulations with the proof-of-concept model, we observed that the SIS are the ones that differ most across community types, whereas weakly interacting species do not differ much (Figure 3d). Thus, if our model applies to realworld microbial communities, SIS could play the role of the bi-stable tipping elements described by Lahti and colleagues (Lahti et al., 2014).

The further investigation of mechanisms behind community types and the identification of strongly interacting microbial species constitutes a promising topic for future research.

\section{Conflict of Interest}

The authors declare no conflict of interest.

\section{Acknowledgements}

LL was funded by Academy of Finland (grants 295741; 307127). Research in the JR laboratory is supported by the FWO, IWT, Rega Institute, KU Leuven, VIB and the Horizon 2020 programs. KF is supported by the KU Leuven. Publication fees were partly covered by the Fondation Universitaire de Belgique.

\section{References}

Arumugam M, Raes J, Pelletier E, Le Paslier D, Yamada T, Mende DR et al. (2011). Enterotypes of the human gut microbiome. Nature 473: 174-180.
Bashan A, Gibson TE, Friedman J, Carey VJ, Weiss ST, Hohmann EL et al. (2016). Universality of human microbial dynamics. Nature 534: 259-262.

Bucci V, Bradde S, Biroli G, Xavier JB. (2012). Social interaction, noise and antibiotic-mediated switches in the intestinal microbiota. PLoS Comput Biol 8: e1002497.

Chatelier EL, Nielsen T, Qin J, Prifti E, Hildebrand F, Falony G et al. (2013). Richness of human gut microbiome correlates with metabolic markers. Nature 500: 541-546.

Connell JH, Sousa WP. (1983). On the evidence needed to judge ecological stability or persistence. Am Nat 121: 789-824.

Costello EK, Stagaman K, Dethlefsen L, Bohannan BJM, Relman DA. (2012). The application of ecological theory toward an understanding of the human microbiome. Science 336: 1255-62.

Dethlefsen L, Relman DA. (2011). Incomplete recovery and individualized responses of the human distal gut microbiota to repeated antibiotic perturbation. Proc Natl Acad Sci USA 108: 4554-4561.

Ding T, Schloss PD. (2014). Dynamics and associations of microbial community types across the human body. Nature 509: 357-360.

Falony G, Joossens M, Vieira-Silva S, Wang* J, Darzi Y, Faust K et al. (2016). Population-level analysis of gut microbiome variation. Science 352: 560-564.

Faust K, Lahti L, Gonze D, de Vos W, Raes J. (2015). Metagenomics meets time series analysis: unraveling microbial community dynamics. Curr Opin Microbiol 25: 56-66.

Gajer P, Brotman RM, Bai G, Sakamoto J, Schuette UME, Zhong $\mathrm{X}$ et al. (2012). Temporal dynamics of the human vaginal microbiota. Sci Transl Med 4: 132ra152.

Gardner TS, Cantor CR, Collins JJ. (2000). Construction of a genetic toggle switch in Escherichia coli. Nature 403: 339-342.

Gibson TE, Bashan A, Cao H-T, Weiss ST, Liu Y-Y. (2016). On the origins and control of community types in the human microbiome. PLoS Comput Biol 12: e1004688.

Gorvitovskaia A, Holmes SP, Huse SM. (2016). Interpreting prevotella and bacteroides as biomarkers of diet and lifestyle. Microbiome 4: 15.

Hirota M, Holmgren M, Van Nes E, Scheffer M. (2011). Global resilience of tropical forest and savanna to critical transitions. Science 334: 232-235.

Knights D, Ward TL, McKinlay CE, Miller H, Gonzalez A, McDonald D et al. (2014). Rethinking 'enterotypes'. Cell Host Microbe 16: 433-437.

Koopman J, Röling W, Buijs M, Sissons C, ten Cate J, Keijser B et al. (2015). Stability and resilience of oral microcosms toward acidification and Candida outgrowth by arginine supplementation. Microb Ecol 69: 422-433.

Lahti L, Salojärvi J, Salonen A, Scheffer M, Vos WMd. (2014). Tipping elements in the human intestinal ecosystem. Nat Commun 5: 4344.

Lewontin RC. (1969). The meaning of stability. Brookhaven Symp Biol 22: 13-24.

May RM. (1977). Thresholds and breakpoints in ecosystems with a multiplicity of stable states. Nature 269: 471-477.

Ravel J, Gajer P, Abdob Z, Schneider GM, Koenig SSK, McCulle SL et al. (2011). Vaginal microbiome of 
reproductive-age women. Proc Natl Acad Sci USA 108 4680-4687.

Rosindell J, Hubbell SP, Etienne RS. (2011). The unified neutral theory of biodiversity and biogeography at age ten. Trends Ecol Evol 26: 340-348.

Scheffer M, Bascompte J, Brock WA, Brovkin V, Carpenter SR, Dakos V et al. (2009). Early-warning signals for critical transitions. Nature 461: 53-59.

Schröder A, Persson L, Roos AMD. (2005). Direct experimental evidence for alternative stable states: a review. OIKOS 110: 3-19.

Shetty SA, Hugenholtz F, Lahti L, Smidt H, Vos WMd. (2017). Intestinal microbiome landscaping: insight in community assemblage and implications for microbial modulation strategies. FEMS Microbiol Rev 41: 182-199.

Stein RR, Bucci V, Toussaint NC, Buffie CG, Raetsch G, Pamer EG et al. (2013). Ecological modeling from time-series inference: insight into dynamics and stability of intestinal microbiota. PLoS Comput Biol 9: e1003388.

Thomas R, D'Ari R. (1990). Biological Feedback. CRC Press: Boca Raton, Florida.

Vieira-Silva S, Falony G, Darzi Y, Lima-Mendez G, Yunta RG, Okuda S et al. (2016). Species-function relationships shape ecological properties of the human gut microbiome. Nat Microbiol 1: 16088.

$\mathrm{Wu}$ GD, Chen J, Hoffmann C, Bittinger K, Chen Y-Y, Keilbaugh SA et al. (2011). Linking long-term dietary patterns with gut microbial enterotypes. Science 334: 105-108.

(c) (1) (3) This work is licensed under a Creative Commons Attribution-NonCommercialShareAlike 4.0 International License. The images or other third party material in this article are included in the article's Creative Commons license, unless indicated otherwise in the credit line; if the material is not included under the Creative Commons license, users will need to obtain permission from the license holder to reproduce the material. To view a copy of this license, visit http://creativecommons.org/ licenses/by-nc-sa/4.0/

(C) The Author(s) 2017

Supplementary Information accompanies this paper on The ISME Journal website (http://www.nature.com/ismej) 